\title{
Hypoxia-activated small molecule-induced gene expression
}

Sarah L. Collins, ${ }^{1,2}$ Jaideep Saha, ${ }^{1,3}$ Laure C. Bouchez, ${ }^{4}$ Ester M. Hammond ${ }^{2 *}$ and Stuart J. Conway ${ }^{1 *}$

1Department of Chemistry, Chemistry Research Laboratory, University of Oxford, Mansfield Road, Oxford, OX1 3TA, UK.

${ }^{2}$ Cancer Research UK/MRC Oxford Institute for Radiation Oncology, Department of Oncology, University of Oxford, Old Road Campus Research Building, Oxford, OX3 7DQ, UK.

${ }^{3}$ Current address: Centre of Biomedical Research, Division of Molecular Synthesis and Drug Discovery, SGPGIMS Campus, Raebareli Road, Lucknow 226014, India ${ }^{4}$ Novartis Institutes for BioMedical Research, Chemical Biology and Therapeutics, Fabrikstrasse 22, 4054 Basel, Switzerland.

${ }^{*}$ corresponding authors

Stuart Conway

Stuart.conway@chem.ox.ac.uk

ORCID - 0000-0002-5148-117X

Ester Hammond

Ester.hammond@oncology.ox.ac.uk

ORCID - 0000-0002-2335-3146 


\section{ABSTRACT}

Hypoxia, conditions of reduced oxygen, occur in a wide variety of biological contexts, including solid tumours and bacterial biofilms, which are relevant to human health. Consequently, the development of chemical tools to study hypoxia is vital. Here we report a hypoxia-activated small molecule-mediated gene expression system using a bioreductive prodrug of the inducer isopropyl 1-thio- $\beta$-D-galactopyranoside (IPTG). As a proof-of-concept we have placed the production of a green fluorescent protein under the control of hypoxia. Our system has the potential to be extended to regulate the production of any given protein of choice. 
Hypoxia, conditions of reduced oxygen, occur in a wide variety of biological environments, including disease-relevant situations such as solid tumours and bacterial biofilms. ${ }^{1,2}$ Hypoxia in solid tumours is caused by their high metabolic demand and abnormal vasculature, resulting in reduced oxygen supply to regions of the tumour, either acutely or chronically. Hypoxic tumours are associated with aggressive tumour phenotypes, increased resistance to radio- and chemotherapy, and, consequently, negatively impact cancer patient prognosis. ${ }^{3}$ In bacteria, decreased oxygen concentrations result in increased biofilm formation, ${ }^{4,5}$ and as drugs cannot penetrate to their core, hypoxic biofilms are more resistant to antibiotic treatment. ${ }^{6}$ Given the problems caused by hypoxia in a number of areas that are of fundamental importance for human health, including cancer therapies and antibiotic resistance, it is clear that chemical tools to study hypoxia are vital. We have previously developed tools to $\operatorname{target}^{7,8}$ and image $^{9}$ hypoxia, which function by exploiting the reducing chemical environment that is characteristic of hypoxia.

One example of such tools are hypoxia-activated prodrugs (HAPs) that offer the ability to release biologically-active compounds preferentially in hypoxia, through attachment of a group that reacts preferentially in this environment. ${ }^{10} \mathrm{~A}$ number of bioreductive moieties, including nitroaryl groups, quinones, and $N$-oxides have been employed in HAPs. $^{7,11-15}$ In the case of nitroaryl-based bioreductive compounds, oxygendependent nitroreductase enzymes catalyse reduction of the nitro group to form a radical anion, which is rapidly re-oxidised back to the nitro group in the presence of oxygen. Under hypoxic conditions, this futile redox cycling does not occur and instead the radical anion is further reduced to form a hydroxylamine or amine; subsequent fragmentation releases the drug (Figure 2A). To date, HAPs have primarily been applied to release non-specific toxins, ${ }^{16}$ but also more recently molecularly targeted 
drugs. ${ }^{7,17,18}$ Here we extend the concept to small molecule-mediated gene expression using the inducer isopropyl 1-thio- $\beta$-D-galactopyranoside (IPTG) (Figure 1). In nature, oxygen-dependent gene expression is achieved through the hypoxia-inducible factor (HIF $)^{19-21}$ system. Our technology can therefore be considered an artificial HIF system, with the additional potential to alter the hypoxia-sensing group to control the degree of hypoxia required for, and the kinetics of, gene induction.

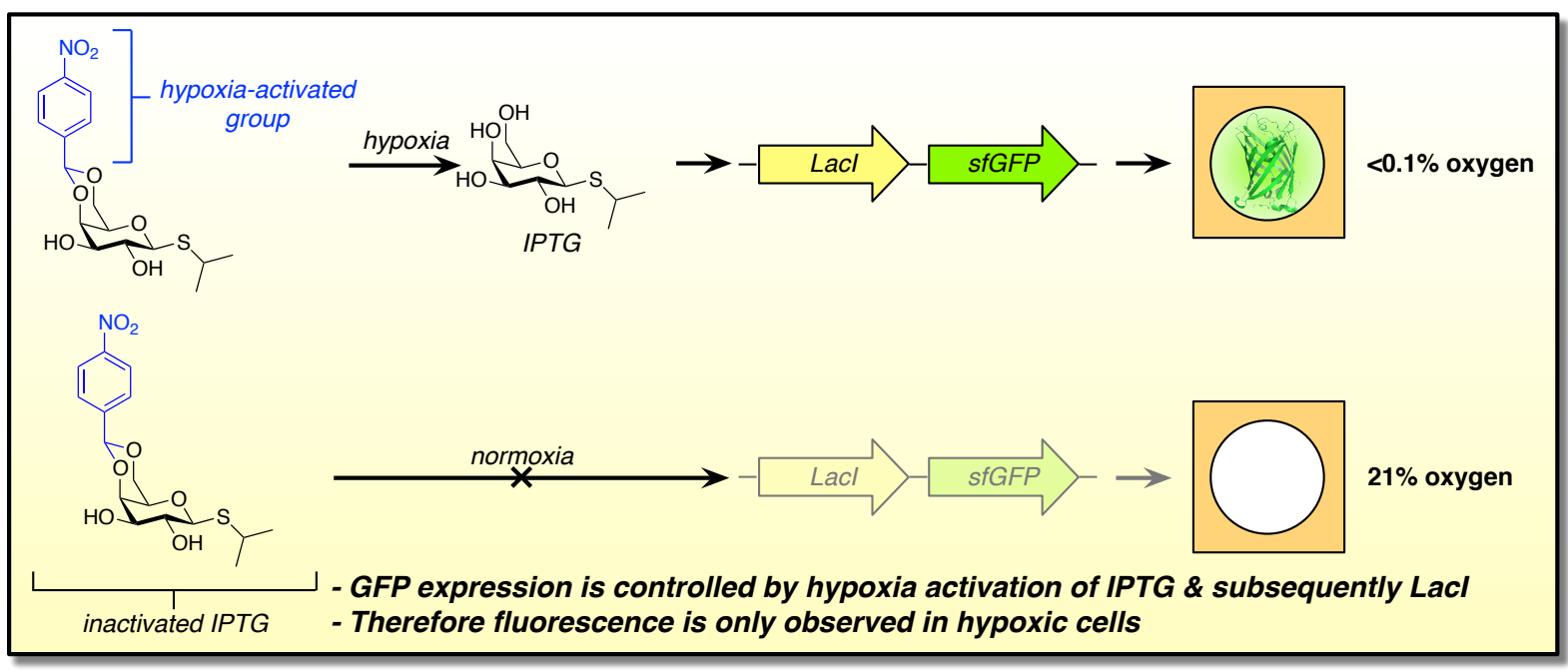

Figure 1. The concept of using hypoxia-activated IPTG to place the lac repressor, and hence gene expression, under the control of hypoxia. 
A
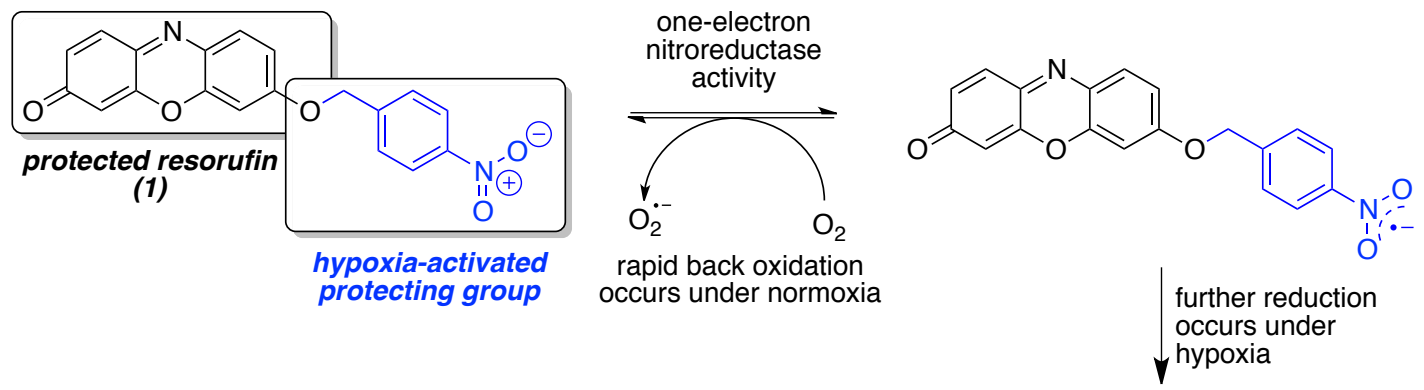<smiles>O=c1ccc2nc3ccc([O-])cc3oc-2c1</smiles>

resorufin, 2 (fluorescent)
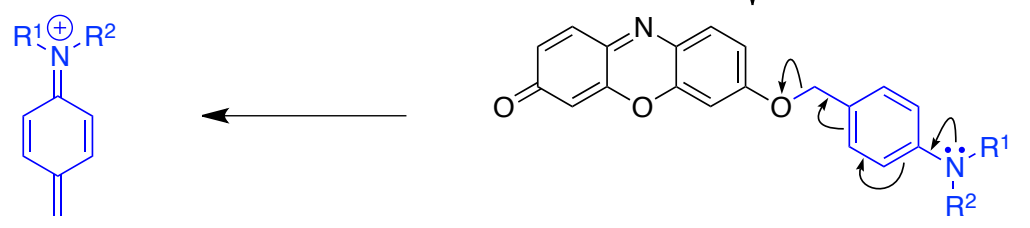

$\mathrm{R}^{1}=\mathrm{R}^{2}=\mathrm{O}$ (nitroso)

$\mathrm{R}^{1}=\mathrm{OH}, \mathrm{R}^{2}=\mathrm{H}$ (hydroxylamine) $\mathrm{R}^{1}=\mathrm{R}^{2}=\mathrm{H}$ (amine)

B<smiles>O=c1ccc2nc3ccc(OCc4ccc([N+](=O)[O-])o4)cc3oc-2c1</smiles>

D

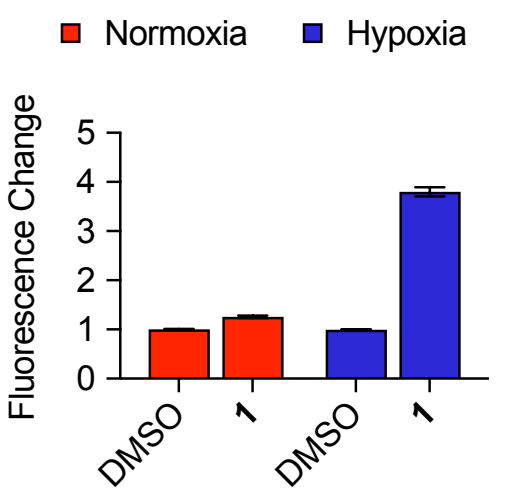

E

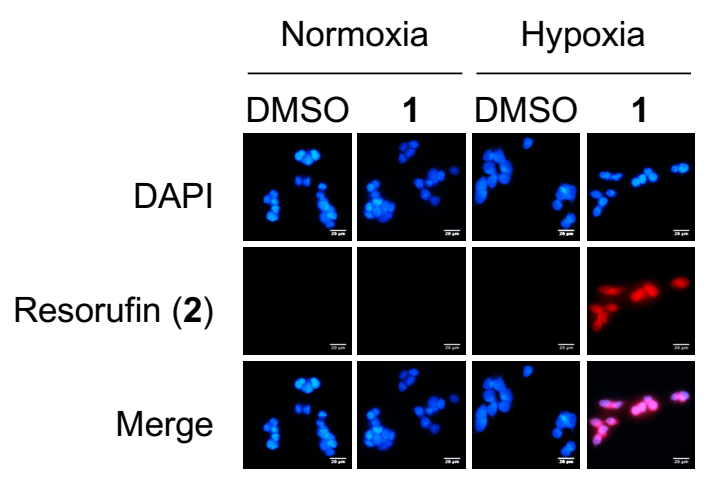

C

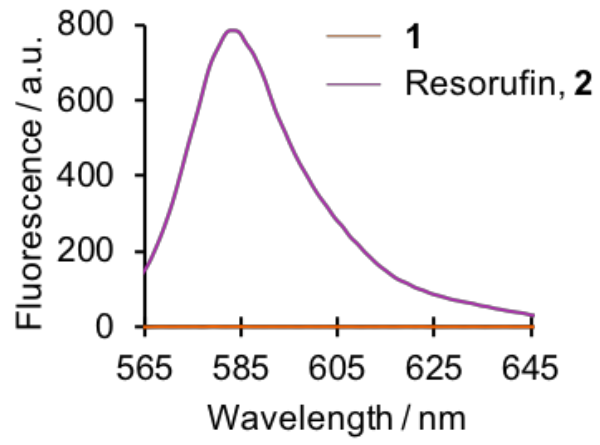

$\boldsymbol{F}$

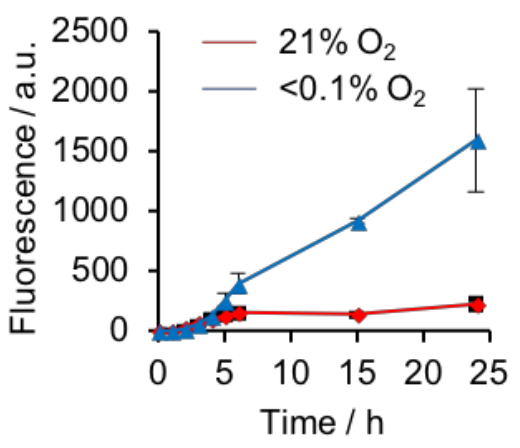

Figure 2. A) The concept of bioreductive fluorescence activation. Attachment of the 4-nitrobenzyl group to the 7-hydroxyl of resorufin affords a non-fluorescent 
compound (1). Under hypoxic conditions, the nitro group is reduced to form an electron-donating substituent, which induces fragmentation and ejects resorufin, which can be detected by fluorescence. B) Structure of the resorufin-based probe 3. C) Fluorescence spectra for resorufin, $2(0.5 \mu \mathrm{M}$ in PBS) and $\mathbf{1}(1 \mu \mathrm{M}$ in PBS); $\lambda_{\mathrm{ex}}=550 \mathrm{~nm}$. D) Quantification of pixel intensities and E) representative images of HCT116 cells $\left(8 \times 10^{5}\right)$ treated with $1(5 \mu \mathrm{M})$ under normoxic $\left(21 \% \mathrm{O}_{2}\right)$ and hypoxic $\left(<0.1 \% \mathrm{O}_{2}\right)$ conditions for $24 \mathrm{~h}$. Cells were fixed and observed by fluorescence microscopy. DAPI (blue) was used as a nuclear stain. Scale bars represent $25 \mu \mathrm{m}$. An increase in fluorescence is only observed in hypoxic conditions. The panels labelled Resorufin (2) show detection of released resorufin and do not indicate addition of resorufin. F) BL21 (DE3) bacteria were treated with $1(5 \mu \mathrm{M})$ under normoxic $\left(21 \% \mathrm{O}_{2}\right)$ and hypoxic $\left(<0.1 \% \mathrm{O}_{2}\right)$ conditions, over $24 \mathrm{~h}$. Samples were analysed by fluorescence $\left(\lambda_{\text {ex: }}: 550 \mathrm{~nm} ; \lambda_{\mathrm{em}}: 585 \mathrm{~nm}\right)$. An increase in fluorescence is only observed in hypoxic conditions.

To demonstrate the principle of molecularly-controlled hypoxia-activated gene expression we needed a system in which a plasmid encoding a protein of our choice could be incorporated. Therefore, we chose to employ bacteria in which the use of plasmids to over-express a protein of choice is well established. For our approach to work, it is necessary that bacteria are able to reduce nitroaryl groups in a hypoxiadependent manner, similar to that observed in mammalian cells. ${ }^{7}$ However, hypoxiadependent bioreduction in bacteria is not well studied. Therefore, to investigate bacterial bioreduction of nitroaromatic-based HAPs, we initially employed the hypoxia-activated fluorescent probe, 3 (Figure 2B). Compound $\mathbf{3}$, comprising the fluorescent dye resorufin (2) conjugated to the bioreductive 5-nitrofuran group, has previously been reported as a switch-on fluorescent probe for hypoxia in mammalian cells. ${ }^{22}$ Alkylation at the 7 -hydroxyl group quenches the fluorescence $\left(\lambda_{550 / 585} \mathrm{~nm}\right)$ of resorufin; this fluorescence can be restored following reduction of the nitro group and subsequent fragmentation, providing a sensitive technique for the detection of hypoxia. However, we observed that in bacteria and mammalian cells this compound underwent bioreduction both in $<0.1 \%$ and $21 \% \mathrm{O}_{2}$, rendering it inappropriate for these studies (Figure S1). As the 4-nitrobenzyl group typically requires lower oxygen concentrations to be bioreduced, ${ }^{23}$ we hypothesised that the analogous compound, $\mathbf{1}$, 
comprising this group attached to the resorufin dye, would be more suitable for our studies (Figure 2A). Fluorescence analysis confirmed that 1 exhibits negligible fluorescence compared to free resorufin (Figure 2C) and analysis in our previously developed sequence of reduction and bioreduction assays ${ }^{23}$ demonstrated it to be suitable for use in a cellular setting (see SI for more details).

Next, compound 1 was used to determine whether hypoxia-dependent bioreduction of a nitroaryl group would take place in bacteria using the $B L 21$ (DE3) strain of Escherichia coli. For comparison, we used the HepG2 liver and HCT116 breast human cancer cell lines, which were also employed in our recent studies on hypoxia imaging. ${ }^{9}$ After 24 hours of incubating 1 in E. coli at either $21 \% \mathrm{O}_{2}$ or $<0.1 \% \mathrm{O}_{2}$, a 7 -fold increase in fluorescence was observed in $<0.1 \% \mathrm{O}_{2}$, compared to the bacteria in $21 \% \mathrm{O}_{2}$ (Figure 2F). Incubation of compound 1 with either HCT116 or HepG2 cells, resulted in an increase in average fluorescence of 3.8-fold and 1.5-fold, respectively, between $21 \% \mathrm{O}_{2}$ and $<0.1 \% \mathrm{O}_{2}$ (Figure 2D/E and Figure S3). Together, these data indicate that hypoxia-dependent bioreduction of $\mathbf{1}$, and subsequent release of resorufin, occur in bacteria in a manner that is similar to that observed in human cells. Consequently, we hypothesised that other HAPs would also function in bacteria.

One of the most commonly used systems to control gene expression in bacteria is based on Lacl. In the absence of an inducer, the lac repressor (Lacl) binds to the DNA, preventing RNA polymerase from initiating transcription. Once added, the IPTG inducer binds to Lacl, causing dissociation from DNA, resulting in transcription and subsequent translation of the downstream gene. ${ }^{24}$ We hypothesised that protecting IPTG with a bioreductive group could inhibit binding of IPTG to Lacl, removing its biological activity. Bioreduction and fragmentation will release IPTG, resulting in 
transcription and translation and therefore placing the downstream gene or genes under the control of hypoxia.

Analysis of a 2.0 A resolution X-ray crystal structure of IPTG bound to the lac repressor [PDB ID: 2P9H $]^{25}$ indicates that all of the hydroxyl groups of IPTG form hydrogen bonding interactions with the protein, either directly or through water molecules (Figure 3A). ${ }^{25}$ It was therefore unclear which was the optimal position for inclusion of the bioreductive group. Consequently, we designed four 4-nitrobenzyl derivatives of IPTG with the bioreductive group attached to each of the 2-, 3-, 4- and 6-position hydroxyl groups (Figure 3B). To facilitate HPLC detection of the compounds we synthesised the corresponding analogues of IPTG with an anomeric benzyl ether that possesses a chromophore for use in the chemical and enzymatic reduction assays (see SI for details). For cellular experiments, the more potent and metabolically stable inducer, IPTG, was used.

A

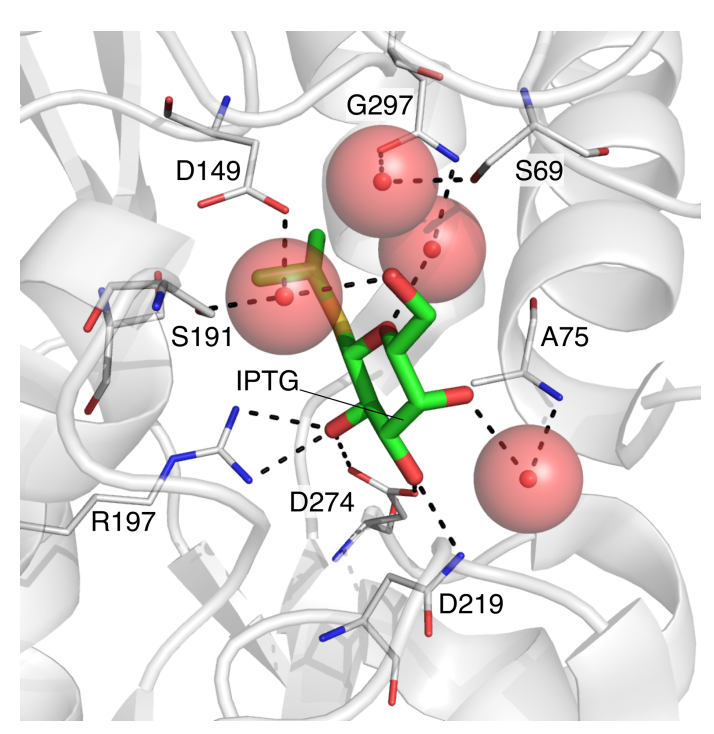

B

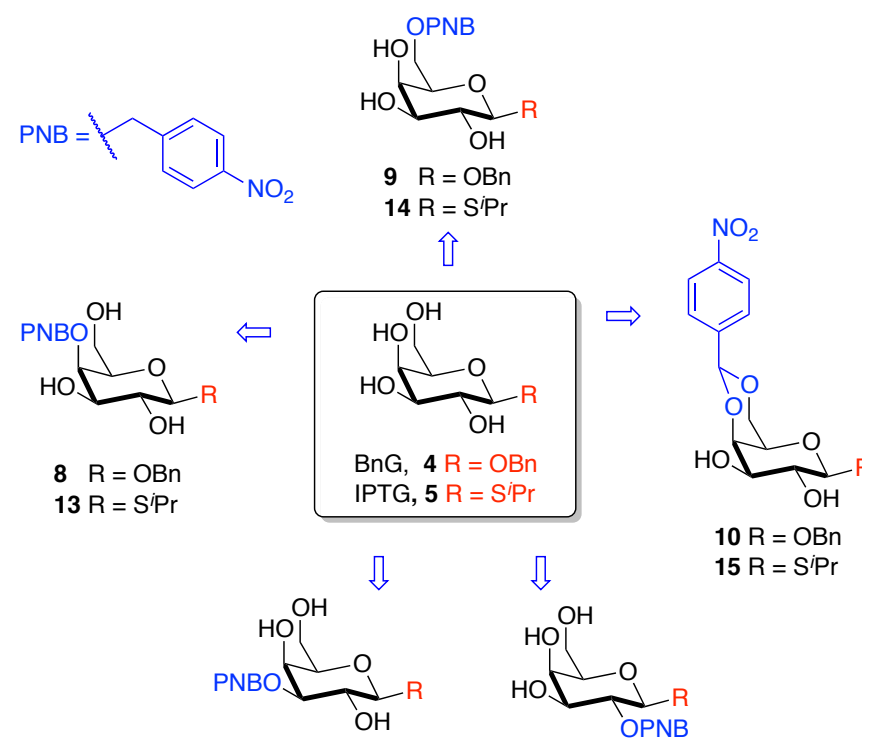

$7 \mathrm{R}=\mathrm{OBn}$ $12 \mathrm{R}=\mathrm{S}^{\mathrm{P} P r}$
$6 \mathrm{R}=\mathrm{OBn}$ $11 \mathrm{R}=\mathrm{S}^{\mathrm{P}} \mathrm{Pr}$

Figure 3. Design of potential bioreductive inducers of gene expression. A) X-Ray crystal structure of IPTG bound to the $E$. coli (K12) lac repressor, showing important hydrogen bonding interactions (PDB code $2 \mathrm{P} 9 \mathrm{H}) .{ }^{25}$ Figure generated using PyMOL. B) Synthetic BnG/IPTG derivatives, 6-15, protected with a 
hypoxia-activated 4-nitrobenzyl group, targeted as potential hypoxia-activated inducers of Lacl.

The syntheses of the 2-, 3-, 4- and 6-position protected 1-O-benzylgalactopyranose derivatives 6-9 and the IPTG derivatives 11-15 are described in detail in the SI. In addition to these ether derivatives, an additional class of HAPs, containing a bioreductive group formed via an acetal linkage between 4- and 6-hydroxyl groups, was also synthesised. These compounds are based on a previously reported photo-activated IPTG derivative. ${ }^{26}$ The 4,6-O-(4-nitrobenzylidene) galactose derivatives were readily synthesised in one step from the corresponding free sugar and 4-nitrobenzaldehyde dimethyl acetal, affording 10 and 15 (Scheme S12).

With these compounds in hand, their ability to undergo bioreduction was assessed in our standard series of assays. We applied the sequence of bioreductive prodrug analysis steps to assess the bioreductive activation of each 4-nitrobenzyl protected compound (6-10). ${ }^{23}$ Firstly, each compound was reduced with zinc and ammonium chloride, forming a mixture of the nitroso- and hydroxylamine derivatives. In each case when an aliquot, taken after 60 minutes of reduction, was added to phosphate buffer, the hydroxylamine derivative fragmented to release the free sugar, 4 (Figure S5), showing that fragmentation was occurring once the nitro group was reduced. We next treated each compound (6-10) with NADPH:P450 reductase enzymes in phosphate buffer to assess their enzymatic reduction. All compounds (6-10) were stable in $21 \% \mathrm{O}_{2}$, while in $<0.1 \% \mathrm{O}_{2}$ reduction to the hydroxylamine and amine derivatives was observed, followed by fragmentation releasing 10-20\% 4. When compound 10 was tested at a concentration of $10 \mu \mathrm{M}$, negligible release of 4 was detected. This suggests that, in this enzyme assay, the acetal is a less effective substrate for the enzyme, or that the compound fragments more slowly than compounds 6-9. To overcome this 
detection problem, and to determine whether any fragmentation was occurring, compound 10 was assessed at a concentration of $20 \mu \mathrm{M}$. The differing amounts of each compound released can be attributed to the difference in enzyme activity on these substrates (Figure 4), as the leaving group ability of each of the hydroxyl groups, and hence propensity of the compound to fragment, is very similar. The lower amount of release from compound $\mathbf{1 0}$ could also be attributed to slower release from the acetal system.

Having confirmed the hypoxia-dependent reduction and fragmentation of these compounds, we next tested their ability to selectively induce gene expression. A superfolder green fluorescent protein (sfGFP), which is a fast-folding version of GFP, ${ }^{27}$ was chosen as the reporter of gene expression, and inserted downstream of an IPTG inducible promoter in the pNIC28-Bsa4 expression vector. ${ }^{28}$ The resulting plasmid was transformed into BL21 (DE3) E. coli. Transformed bacteria were incubated with each compound, in either $21 \%$ or $<0.1 \% \mathrm{O}_{2}$, for 24 hours and the compounds were assessed for their ability to induce gene expression by both fluorescence analysis and western blot. If the 4-nitrobenzyl group inhibits binding to the lac repressor no GFP expression will be observed in $21 \% \mathrm{O}_{2}$, while in $<0.1 \% \mathrm{O}_{2}$, fragmentation to release IPTG will induce GFP expression and consequently an increase in fluorescence will be observed. 

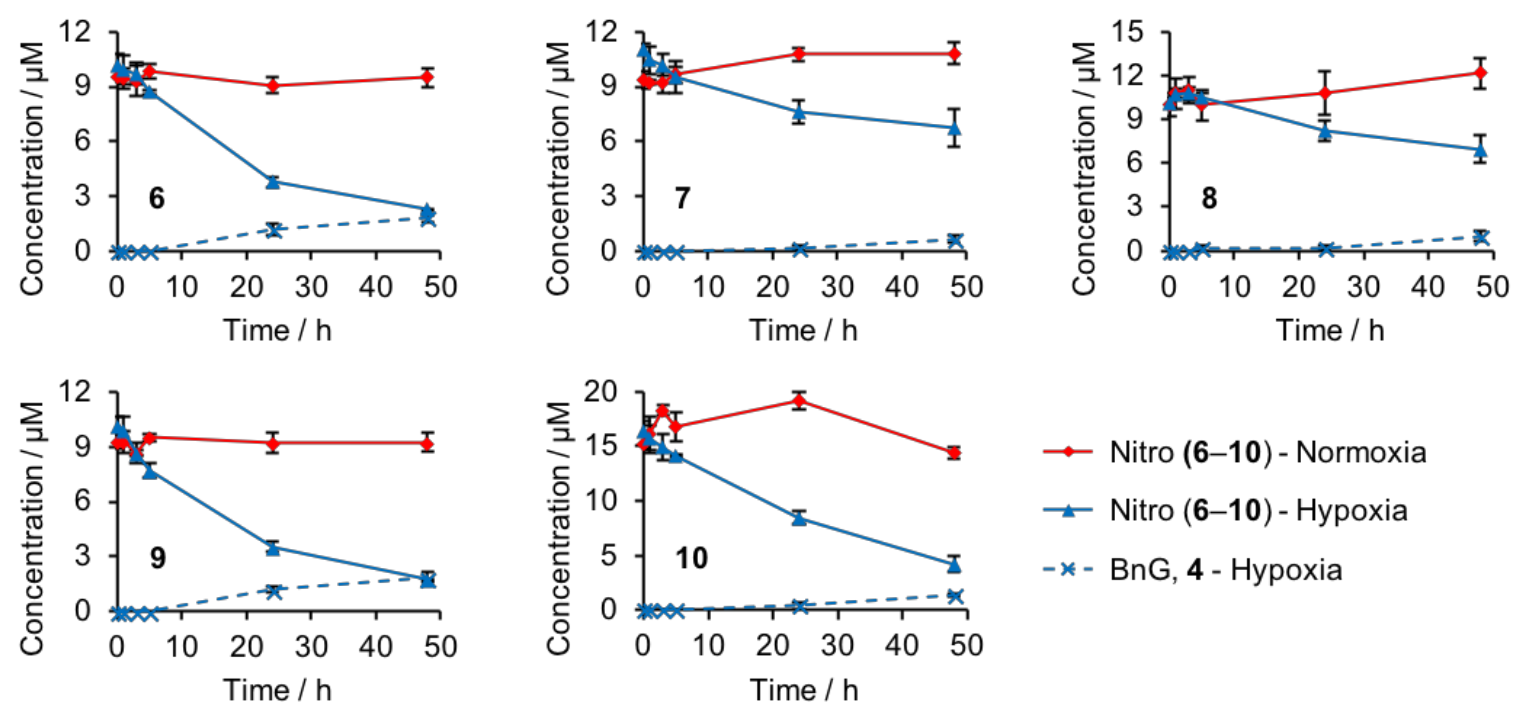

$\rightarrow$ Nitro (6-10) - Normoxia

$\leftarrow$ Nitro (6-10) - Hypoxia

-× - BnG, 4 - Hypoxia

Figure 4. Hypoxia-activated IPTG derivatives are reduced selectively under hypoxia. Compounds 6-9 $(10 \mu \mathrm{M})$ and $10(20 \mu \mathrm{M})$ were treated with NADPH:P450 reductase enzymes under normoxic $\left(21 \% \mathrm{O}_{2}\right)$ and hypoxic $\left(<0.1 \% \mathrm{O}_{2}\right)$ conditions over $48 \mathrm{~h}$. Aliquots were taken at the time indicated and analysed by HPLC.

Western blot analysis and fluorescence studies (Figure 5A-D) showed that 14 induced sfGFP expression under both normoxic and hypoxic conditions. This result indicates that the 4-nitrobenzyl group can be accommodated by Lacl at the 6-position and does not inhibit binding of IPTG to the promoter. Inspection of the X-ray crystal structure shows that the 6-position of IPTG oriented towards a pocket that is likely occupied by the second saccharide ring of allolactose, which is the endogenous ligand for Lacl. The PNB-ether derivatives 11, 12, and 13 showed no induction of GFP expression in $21 \% \mathrm{O}_{2}$, consistent with the X-ray crystal structure that indicated substitutions would not be tolerated. However, none of these compounds caused significant increase in GFP expression in $<0.1 \% \mathrm{O}_{2}$, indicating that significant quantities of IPTG are not being released (Figure 5A-D). This is consistent with the data obtained in the enzyme assay, which also showed that low levels of the parent compound (6-8) were released. In contrast, the acetal 15, which also showed no fluorescence at $21 \% \mathrm{O}_{2}$, displayed a substantial increase in 
fluorescence at $<0.1 \% \mathrm{O}_{2}$ with a corresponding increase in the amount of sfGFP detected by western blot; no sfGFP was detected in normoxia (Figure 5A-D). Cellular imaging experiments (Figure 5E) also show that 15 does not induce sfGFP expression in BL21 (DE3) pNIC28-sfGFP in $21 \% \mathrm{O}_{2}$, but significant expression of sfGFP is observed in $<0.1 \% \mathrm{O}_{2}$. Quantification of the western blot shown in Figure 5C (Figure S7) demonstrates that more protein is produced than indicated by fluorescence analysis (Figure 5A and B), which we attribute to slower formation of the sfGFP chromophore in hypoxia. ${ }^{29}$ To determine the rate of activation of gene expression we carried out a time course experiment, which showed that after an initial lag, hypothesised to be caused by the initial activation step required to release IPTG, sfGFP expression rapidly increased with time until saturation was reached after 24 hours. Importantly, this lag was not observed when free IPTG was added, consistent with this hypothesis (Figure 5G). Finally, we also investigated the oxygen dependency of the bioreductive activation. While no GFP expression was observed at $1 \% \mathrm{O}_{2}$, there was partial induction at $0.5 \% \mathrm{O}_{2}$ and high levels of expression at $0.1 \% \mathrm{O}_{2}$, demonstrating the oxygen dependency of the response (Figure $5 \mathrm{~F}$ ). 

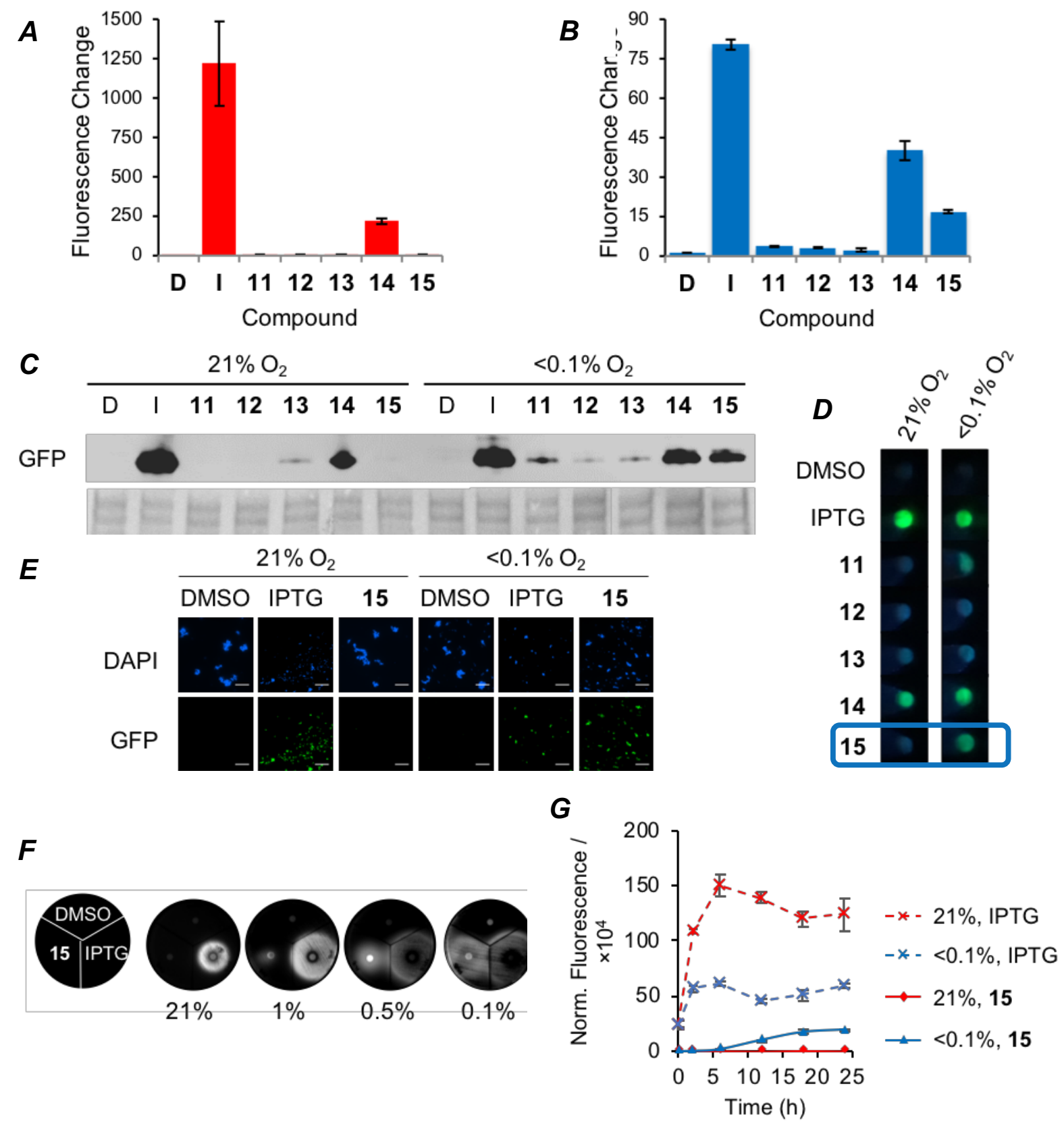

Figure 5. Compound 15 induces gene expression selectively in hypoxia in BL21 (DE3) bacteria. A) BL21 (DE3) pNIC28-sfGFP were treated with DMSO (D), IPTG (I) or hypoxia-activated IPTG derivatives $(1 \mathrm{mM})$ for $24 \mathrm{~h}$ under normoxic $\left(21 \% \mathrm{O}_{2}\right)$ conditions. Samples were analysed by fluorescence $\left(\lambda_{\mathrm{ex}}: 485 \mathrm{~nm}, \lambda_{\mathrm{em}}\right.$ : $520 \mathrm{~nm}$, PBS). Fluorescence was divided by the optical density at $600 \mathrm{~nm}$ and normalised to the relevant DMSO control to account for the higher baseline expression observed for the hypoxia DMSO samples. IPTG and 14 showed an increase in fluorescence under these conditions. B) Conditions as in (A), except treatment under hypoxic $\left(<0.1 \% \mathrm{O}_{2}\right)$ conditions. Both compound 14 and 15 show an increase in fluorescence, indicating that compound 15 is able to induce GFP production selectively in hypoxia. C) Conditions as in (A/B). Samples were analysed by western blot. Ponceau staining is shown as a loading control (see Figure S7 for quantification of band intensity and for the full Ponceau stained membrane). The results mirror those observed by fluorescence. D) Conditions as in (A/B). Bacterial samples were pelleted and imaged under a UV lamp ( $\lambda_{\text {ex: }}$ : 
$365 \mathrm{~nm}$ ), showing the hypoxia-dependent production of GFP promoted by 15. E) Conditions as in (A/B). Samples were suspended in PBS and visualised by fluorescence microscopy. DAPI (blue) was used as a nucleic acid stain. Scale bars $=25 \mu \mathrm{M} . \mathrm{F}$ ) Oxygen dependency of hypoxia-activated gene expression. BL21 (DE3) $p N I C 28-s f G F P$ were spread on $2 \times$ YT agar plates. A disc coated with DMSO, IPTG, or 15 (10 mM) was placed in each third. The plates were incubated at the required oxygen tension for $24 \mathrm{~h}$ and then imaged by fluorescence. $\mathrm{G})$ Time course analysis of IPTG and 15. BL21 (DE3) pNIC28-sfGFP were treated with IPTG or $15(1 \mathrm{mM})$ under $21 \% \mathrm{O}_{2}$ and $<0.1 \% \mathrm{O}_{2}$ conditions over 24 hours. Samples, taken at the time points shown were analysed by fluorescence $\left(\lambda_{\text {ex: }}\right.$ : $\left.485 \mathrm{~nm}, \lambda_{\mathrm{em}}: 520 \mathrm{~nm}, \mathrm{PBS}\right)$. Fluorescence was divided by the optical density at $600 \mathrm{~nm}$.

It is interesting to note that, while the enzyme-based assays show similar release of IPTG from both the nitrobenzyl ether and acetal-based compound (15), the latter shows significantly more induction of sfGFP expression in a cellular setting. To our knowledge, there are no previous examples of acetal-based bioreductive groups, and it seems reasonable that this structural difference must account for the higher activation observed in cells. We propose a putative mechanism (Figure 6), which involved reduction to the corresponding amine, followed by two pathways for the initial fragmentation step. These result in extended enol-ether-like systems attached to either the 4- or the 6-position. We propose that these systems are intercepted by water or other endogenous nucleophiles (e.g. GSH) to give a hemi-acetal (or equivalent), which collapses to release IPTG and 4-aminobenzaldehyde (21). It is possible that the involvement of external nucleophiles promotes this mechanism in cells. HPLC analysis of 10 treated with NADPH:P450 reductase enzymes under $21 \% \mathrm{O}_{2}$ and $<0.1 \% \mathrm{O}_{2}$, revealed that 4-aminobenzaldehyde (21) was only observed in the $<0.1 \% \mathrm{O}_{2}$ (Figure S8), supporting the above mechanism.

As an alternative, we note that compound 14, in which the 4-nitrobenzyl group is attached to the 6-position of IPTG, promoted gene expression in $21 \% \mathrm{O}_{2}$. Therefore, it is feasible that compound $\mathbf{1 8}$, which results from reduction and initial fragmentation is sufficient to induce gene expression. To test this, we synthesised the 
6-aminobenzyl derivative of IPTG (22), an analogue of 18, and incubated it with $B L 21$ (DE3) in either $21 \%$ or $<0.1 \% \mathrm{O}_{2}$ for 24 hours. Induction of GFP expression was observed in both conditions (Figure S9), suggesting that 22 is able to bind to Lacl and induce gene expression. As the anomeric benzyl analogue of compound 22, compound $\mathbf{S 2 8}$, was observed to be stable in phosphate buffer AT $37^{\circ} \mathrm{C}$ for 24 hours (Figure S10), we propose that fragmentation to release IPTG is not required for gene expression. Consequently, it seems that there are two mechanisms by which bioreduction of $\mathbf{1 5}$ can induce gene expression, accounting for its greater activity in bacteria.

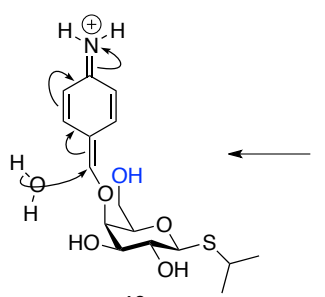

19

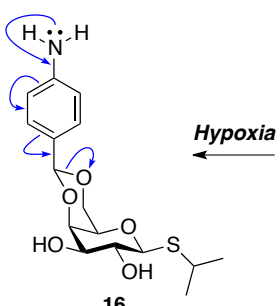

16

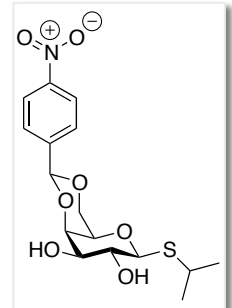

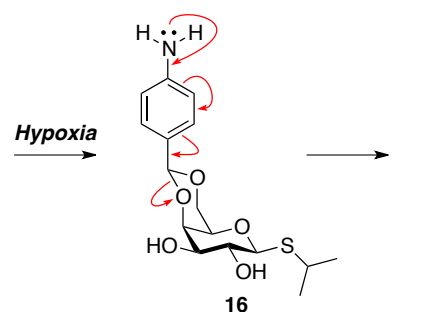

16

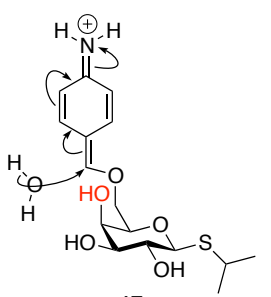

17

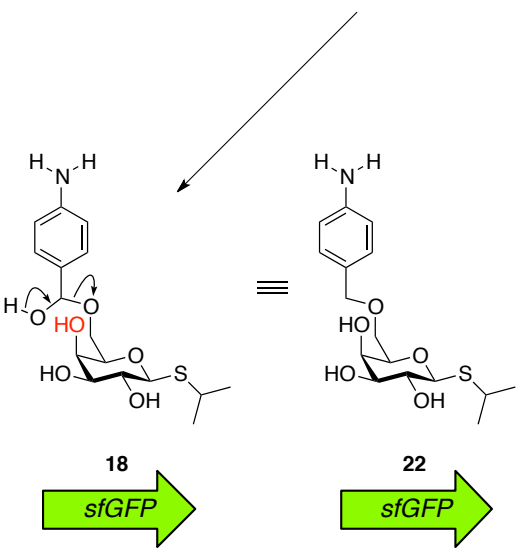

Figure 6. Proposed mechanisms for fragmentation of compound 15 to release IPTG (5) and 4-aminobenzaldehyde (21). It is proposed that, in addition to 5, compound 18 is also able to induce gene expression, as its analogue $\mathbf{2 2}$ induces production of sfGFP.

Taken together, our data demonstrate that we have developed an artificial HIF system, which places genes under the control of a hypoxia-activated inducer. While we have used sfGFP as our reporter, there is no limitation on the genes that we can control in this system. As it stands, our work will allow the investigation of hypoxia- 
activated gene expression in bacteria, which has especially significant implications in the study of biofilms. Future work will focus on transferring this technology into human cells, where although less prevalent, the Lacl promoter system can also be used, ${ }^{30,31}$ and on modification of the bioreduction potential to modulate the rate of IPTG release and induction of gene expression. ${ }^{32} \mathrm{~A}$ third area of application is plants, where genetic modification is commonplace, and this technology could find wide spread use in crop protection and security.

\section{ASSOCIATED CONTENT}

Supporting information including supporting figures, supporting schemes, chemistry experimental details and full details of compounds characterisation, biological methods, NMR spectra for compounds synthesized, is available is available free of charge via the internet at http://pubs.acs.org.

\section{ACKNOWLEDGEMENTS}

S.L.C. and S.J.C. thank the BBSRC and Novartis for the award of a BBSRC iCASE studentship (BB/L016214/1). This project has received funding from the European Union's FP7 research and innovation programme under grant agreement number 331327. E.M.H. is supported by a Cancer Research UK grant (C5255/A23755). S.J.C. thanks St Hugh's College, Oxford, for research funding.

\section{CONFLICTS OF INTEREST}

The authors declare no conflict of interests.

\section{KEYWORDS}


Collins et al.

Hypoxia; prodrugs; bioreduction; gene expression 


\section{REFERENCES}

(1) Höckel, M.; Vaupel, P. Tumor Hypoxia: Definitions and Current Clinical, Biologic, and Molecular Aspects. J. Natl. Cancer Inst. 2001, 93, 266-276.

(2) Rossignol, T.; Ding, C.; Guida, A.; d'Enfert, C.; Higgins, D. G.; Butler, G. Correlation between Biofilm Formation and the Hypoxic Response in Candida Parapsilosis. Eukaryot. Cell 2009, 8, 550-559.

(3) Brown, J. M.; Wilson, W. R. Exploiting Tumour Hypoxia in Cancer Treatment. Nat. Rev. Cancer 2004, 4, 437-447.

(4) Cramton, S. E.; Ulrich, M.; Götz, F.; Döring, G. Anaerobic Conditions Induce Expression of Polysaccharide Intercellular Adhesin in Staphylococcus Aureus and Staphylococcus Epidermidis. Infect. Immun. 2001, 69, 4079-4085.

(5) Mashruwala, A. A.; Guchte, A. van de; Boyd, J. M. Impaired Respiration Elicits SrrAB-Dependent Programmed Cell Lysis and Biofilm Formation in Staphylococcus Aureus. eLife 2017, 6, e23845. https://doi.org/10.7554/eLife.23845.

(6) Borriello, G.; Werner, E.; Roe, F.; Kim, A. M.; Ehrlich, G. D.; Stewart, P. S. Oxygen Limitation Contributes to Antibiotic Tolerance of Pseudomonas Aeruginosa in Biofilms. Antimicrob. Agents Chemother. 2004, 48, 2659-2664.

(7) Cazares-Körner, C.; Pires, I. M.; Swallow, I. D.; Grayer, S. C.; O’Connor, L. J.; Olcina, M. M.; Christlieb, M.; Conway, S. J.; Hammond, E. M. CH-01 Is a Hypoxia-Activated Prodrug That Sensitizes Cells to Hypoxia/Reoxygenation Through Inhibition of Chk1 and Aurora A. ACS Chem. Biol. 2013, 8, 1451-1459.

(8) O'Connor, L. J.; Cazares-Körner, C.; Saha, J.; Evans, C. N. G.; Stratford, M. R. L.; Hammond, E. M.; Conway, S. J. Efficient Synthesis of 2-Nitroimidazole Derivatives and the Bioreductive Clinical Candidate Evofosfamide (TH-302). Org. Chem. Front. 2015, 2, 1026-1029.

(9) O’Connor, L. J.; Mistry, I. N.; Collins, S. L.; Folkes, L. K.; Brown, G.; Conway, S. J.; Hammond, E. M. CYP450 Enzymes Effect Oxygen-Dependent Reduction of Azide-Based Fluorogenic Dyes. ACS Cent. Sci. 2017, 3, 20-30.

(10) Wilson, W. R.; Hay, M. P. Targeting Hypoxia in Cancer Therapy. Nat. Rev. Cancer 2011, 11, 393410.

(11) Peters, K. B.; Brown, J. M. Tirapazamine. Cancer Res. 2002, 62, 5248-5253. 
(12) McKeown, S.; Hejmadi, M.; Mclntyre, I.; McAleer, J.; Patterson, L. AQ4N: An Alkylaminoanthraquinone N-Oxide Showing Bioreductive Potential and Positive Interaction with Radiation in Vivo. Br. J. Cancer 1995, 72, 76-81.

(13) Duan, J.-X.; Jiao, H.; Kaizerman, J.; Stanton, T.; Evans, J. W.; Lan, L.; Lorente, G.; Banica, M.; Jung, D.; Wang, J.; Ma, H.; Li, X.; Yang, Z.; Hoffman, R. M.; Ammons, W. S.; Hart, C. P.; Matteucci, M. Potent and Highly Selective Hypoxia-Activated Achiral Phosphoramidate Mustards as Anticancer Drugs. J. Med. Chem. 2008, 51, 2412-2420.

(14) Blanche, E. A.; Maskell, L.; Colucci, M. A.; Whatmore, J. L.; Moody, C. J. Synthesis of Potential Prodrug Systems for Reductive Activation. Prodrugs for Anti-Angiogenic Isoflavones and VEGF Receptor Tyrosine Kinase Inhibitory Oxindoles. Tetrahedron 2009, 65, 4894-4903.

(15) Fryatt, T.; Pettersson, H. I.; Gardipee, W. T.; Bray, K. C.; Green, S. J.; Slawin, A. M. Z.; Beall, H. D.; Moody, C. J. Novel Quinolinequinone Antitumor Agents: Structure-Metabolism Studies with NAD(P)H:Quinone Oxidoreductase (NQO1). Bioorg. Med. Chem. 2004, 12, 1667-1687.

(16) Liu, J.; Bu, W.; Shi, J. Chemical Design and Synthesis of Functionalized Probes for Imaging and Treating Tumor Hypoxia. Chem. Rev. 2017, 117, 6160-6224.

(17) Granchi, C.; Funaioli, T.; Erler, J. T.; Giaccia, A. J.; Macchia, M.; Minutolo, F. Bioreductively Activated Lysyl Oxidase Inhibitors against Hypoxic Tumours. ChemMedChem 2009, 4, 1590_ 1594.

(18) Mistry, I. N.; Thomas, M.; Calder, E. D. D.; Conway, S. J.; Hammond, E. M. Clinical Advances of Hypoxia-Activated Prodrugs in Combination With Radiation Therapy. Int. J. Radiat. Oncol. 2017, 98, 1183-1196.

(19) Semenza, G. L. HIF-1: Mediator of Physiological and Pathophysiological Responses to Hypoxia. J. Appl. Physiol. 2000, 88, 1474-1480.

(20) Schofield, C. J.; Ratcliffe, P. J. Oxygen Sensing by HIF Hydroxylases. Nat. Rev. Mol. Cell Biol. 2004, 5, 343-354.

(21) Keith, B.; Johnson, R. S.; Simon, M. C. HIF1a and HIF2a: Sibling Rivalry in Hypoxic Tumour Growth and Progression. Nat. Rev. Cancer 2012, 12, 9-22.

(22) Li, Z.; Li, X.; Gao, X.; Zhang, Y.; Shi, W.; Ma, H. Nitroreductase Detection and Hypoxic Tumor Cell Imaging by a Designed Sensitive and Selective Fluorescent Probe, 7-[(5-Nitrofuran-2YI)Methoxy]-3 H-Phenoxazin-3-One. Anal. Chem. 2013, 85, 3926-3932. 
(23) O'Connor, L. J.; Cazares-Körner, C.; Saha, J.; Evans, C. N. G.; Stratford, M. R. L.; Hammond, E. M.; Conway, S. J. Design, Synthesis and Evaluation of Molecularly Targeted Hypoxia-Activated Prodrugs. Nat. Protoc. 2016, 11, 781-794.

(24) Lewis, M. The Lac Repressor. C. R. Biol. 2005, 328, 521-548.

(25) Daber, R.; Stayrook, S.; Rosenberg, A.; Lewis, M. Structural Analysis of Lac Repressor Bound to Allosteric Effectors. J. Mol. Biol. 2007, 370, 609-619.

(26) Young, D. D.; Deiters, A. Photochemical Activation of Protein Expression in Bacterial Cells. Angew. Chem. Int. Ed. 2007, 46, 4290-4292.

(27) Pédelacq, J.-D.; Cabantous, S.; Tran, T.; Terwilliger, T. C.; Waldo, G. S. Engineering and Characterization of a Superfolder Green Fluorescent Protein. Nat. Biotechnol. 2006, 24, 79-88.

(28) Savitsky, P.; Bray, J.; Cooper, C. D. O.; Marsden, B. D.; Mahajan, P.; Burgess-Brown, N. A.; Gileadi, O. High-Throughput Production of Human Proteins for Crystallization: The SGC Experience. J. Struct. Biol. 2010, 172, 3-13.

(29) Vordermark, D.; Shibata, T.; Brown, J. M. Green Fluorescent Protein Is a Suitable Reporter of Tumor Hypoxia Despite an Oxygen Requirement for Chromophore Formation. Neoplasia 2001, $3,527-534$.

(30) Hu, M. C. T.; Davidson, N. The Inducible lac Operator-Repressor System Is Functional in Mammalian Cells. Cell 1987, 48, 555-566.

(31) Wyborski, D. L.; DuCoeur, L. C.; Short, J. M. Parameters Affecting the Use of the Lac Repressor System in Eukaryotic Cells and Transgenic Animals. Environ. Mol. Mutagen. 1996, 28, 447-458.

(32) Liang, D.; Miller, G. H.; Tranmer, G. K. Hypoxia Activated Prodrugs: Factors Influencing Design and Development. Curr. Med. Chem. 2015, 22, 4313-4325. 